\title{
Doorstep delivery of essential healthcare services during the nationwide COVID-19 lockdown in Bhutan
}

\author{
Dawa Gyeltshen ${ }^{1}$, Thinley Dorji ${ }^{2,3}$
}

\section{AFFILIATION}

1 Eusa Hospital, Ministry of Health, Wangdue Phodrang, Bhutan

2 Department of Internal Medicine, Armed Forces Medical College, Maharashtra University of Health Sciences, Maharashtra, India

3 Kidu Mobile Medical Unit, His Majesty's People's Project, Thimphu, Bhutan

\section{CORRESPONDENCE TO}

Dawa Gyeltshen. Eusa Hospital, Ministry of Health, Wangdue Phodrang, Bhutan.E-mail: waelshey1@gmail.com

\section{KEYWORDS}

essential healthcare services, primary healthcare, COVID-19

Received: 14 September 2020, Revised: 30 October 2020, Accepted: 3 November 2020

\section{Dear Editor,}

Long periods of lockdown due to COVID-19 have compromised the delivery of essential healthcare services including immunization programs in many countries ${ }^{1}$. Bhutan, a small Himalayan nation with a population of about 0.7 million people, imposed its first lockdown for 21 days in August 2020 followed by two weeks of restrictions of movement to contain the spread of COVID-192. By 29 October 2020, there were 346 COVID-19 cases confirmed in Bhutan with a male to female ratio of $2.5: 1$. The recovery rate of COVID-19 cases was 91\% (315) with zero deaths. Since the start of the COVID-19 pandemic till October 2020, Bhutan has carried out 169479 COVID-19 tests with a test rate of 233075 per million population - 81316 (48\%) RTPCR tests and 88163 (52\%) RDT tests ${ }^{3}$. This letter describes the measures taken by the government to ensure continued delivery of essential healthcare services during the lockdown period.

Bhutan has a robust and well-established primary healthcare system with community health workers including doctors, nurses, traditional medicine practitioners and health assistants providing services within three hours walking distance to more than $95 \%$ of the population ${ }^{4}$. All health centers in Bhutan provide primary healthcare services. In 2020, there were 49 hospitals, 186 primary healthcare centers, 53 sub-posts, 542 outreach clinics, and 3 municipal clinics $^{4}$. During the nationwide lockdown, the government implemented emergency measures to ensure uninterrupted delivery of essential healthcare services. The essential healthcare service delivery plan package prioritized mother and child health services including the extended program on immunization, antenatal care, institutional deliveries of babies services, postnatal care, and family planning services. Routine uninterrupted care for patients with noncommunicable diseases, HIV, tuberculosis, and mental health problems, were also provided.

The essential healthcare package of services was delivered at the doorstep of each household through mobile clinics and telemedicine facilities. While deliveries of babies were conducted at health centers to minimize intrapartum and postpartum risks, identified health workers provided domiciliary antenatal and postnatal care for high-risk babies and mothers. Those mothers with low risks were remotely monitored through telephonic communications. Only those healthcare workers with no risk factors for severe COVID-19 infection were allocated the task of mobile service delivery.

Providing uninterrupted routine essential healthcare during COVID-19 lockdown was essential to prevent further loss of lives and to achieve Bhutan's goal of universal healthcare. Bhutan's commitment to primary healthcare has been fundamental in its health success story so far. These measures taken by the government during the nationwide lockdown demonstrate its commitment towards primary healthcare and its goal of achieving universal healthcare.

\section{REFERENCES}

1. Centers for Disease Control and Prevention. Maintaining Essential Health Services During COVID-19 in Low Resource, Non-U.S. Settings. https://www.cdc.gov/coronavirus/2019ncov/global-covid-19/essential-health-services.html. Updated September 18, 2020. Accessed October 30, 2020.

2. Dorji P. Government activates nationwide lockdown. Bhutan Broadcasting Service. http://www.bbs.bt/news/?p=135610. Published August 11, 2020. Accessed October 30, 2020. 
3. Ministry of Health. National Situational Update On COVID-19 (Data As Of 28th October 2020). http://www.moh.gov.bt/ national-situational-update-on-covid-19-data-as-of-28thoctober-2020/?fbclid=IwAR0Emx93Mju2pEN6mQ9zDEKejf qj-mWW65G6mxdYaHYRBgjxBAI9J3BjA_k. Accessed October 30, 2020.

4. Ministry of Health. Annual Health Bulletin 2020. Thimphu: Ministry of Health, Royal Government of Bhutan; 2020. http://www.moh.gov.bt/wp-content/uploads/ictfiles/2017/06/health-bulletin-Website_Final.pdf. Accessed October 30, 2020.

\section{CONFLICTS OF INTEREST}

The authors have completed and submitted the ICMJE Form for Disclosure of Potential Conflicts of Interest and none was reported.

\section{PROVENANCE AND PEER REVIEW}

Not commissioned; internally peer reviewed.

\section{FUNDING}

There was no source of funding for this research. 